113 cases of all 210 administrations into our hospital in the period from June 1964 to August 1965 were emergent (53.8\%), of which head injuries were 86 cases $(76.9 \%)$ and others were only 26 cases $(23.1 \%)$. Of all head injuries 30 cases were operated on, of which 4 cases were died, but 2 cases of these would have been relieved, if the patients were transfered smoothly to our hospital. All head injuries were classified to 51 cases of concussion type, 16 cases of laceration type and 11 cases of hematoma type, and of 11 cases of all acute intracranical hematoma epidual type was 4, subdural type 4, intracerebral type 2 and subdural intracerebral type 1. Analysis in age of patients of acute head injuries showed that the patient of age under 10 year-old was $55.1 \%$ and the patient of age over 40 year-old was rather low percentage. The cause of frequent occurrence of head injuries in the low aged childlen and infants was due to traffic accident and fall from height within the residence, and that especially the traffic accident was $55.1 \%$ of the causes of all head injuries. Of 26 non-traumatic emergency cases cerebrovascular lesion was $38.1 \%$ and this reason of high percentage of cerebrovascular lesion was because of being sent of unconscious patients from other hospital.

\title{
3. Acute Intracranical Hematoma, Report of 113 Cases
}

\author{
Shuro Nishimura, Takashi Nakamura, Tamotsu Asakura, \\ Yoshihiko Umebayashi, Kunihiko Osaka \\ and Kazuo EBINA \\ Neurosurgery, Kitano Hospital, Tazuke Kofukai Medical \\ Research Institute, Osaka
}

Analysis of 113 cases of acute intracranical hematoma (34 extradual hematoma cases, 79 subdural hematoma cases) is reported. Molitality is $27 \%$ in extradural hematoma group and $77 \%$ in subdural hematoma group.

Prognosis of patients having subdural hematoma is quite poor, even if craniotomy is performed in early stage following lucid interval. Simple evacuation of clot alone can not save majority of patients with subdural hematoma. In those cases cause of death is brain swelling which is present with hematoma or appeare after removal of clot. Therefore, treatment of cerebral swelling should be considered not only in severe cases but also in patients with slight consciousness disturbance in conjunction with operative therapy. Inhalation of oxygen, securing of airway, administration 
of stroids and hypertonic solution such as mannitol and urea, and hypothermia should be commenced before operation and continued postperatively.

In several cases of subdural hematoma, showing lucid interval, clot of small amount was noted at operation and cause of conciousness deterioration seemed to be brain swelling. In such cases, conservative treatment is prefered to surgery.

Since improvement in consciousness state was seen after treatment of brain swelling was done in patients having subdural hematoma of moderate size, better result might be obtained by performing operation after consciousness level rises by means of conservative measures in some cases.

In majority of present cases, cerebral angiography was not performed and hematoma was discovered by neurological examination and/or inspection burr hole technique. Since arteriography will be helpfull to know not only location but amount of clot and to determine validity and time of operative intervention in subdural hematoma cases, it is better to make the test before operation, when subdural hematoma is suspected, unless deterioration of vital sign such as respiratory disturbance is present.

\title{
4. Increasing of the Subacute Stage Operations of Posttraumatic Intracranial Hematomas
}

\author{
Toshisuke Yamamoto, Tomoo Kagami and Hisashi Yoshida \\ Department of Surgery, Mie Prefectural University \\ School of Medicine
}

In the last two years cases of subacute intracranial hematomas increased predominantly in our hospital. Before admission in their subacute stage, almost all the patients had been in sensory disturbances, and mannitol, urea or other hypertonic solutions, occasionally combined with synthetic adrenocortical hormones had been administered to them by emergency doctors already in acute period. As generally accepted, the results were good in all the cases operated in subacute stage. General use of the hypertonics and other modern remedies for brain edema seems to be playing an important part as a causative moment of increasing of the subacute intracranical hematomas. Administration of the drugs brings good luck or not, that is the problem. 